\title{
THE FORMS $a x^{2}+b y^{2}+c z^{2}$ WHICH REPRESENT ALL INTEGERS
}

BY L. E. DICKSON

Theorem. $f=a x^{2}+b y^{2}+c z^{2}$ represents all integers, positive, negative, or zero, if and only if: I. $a, b, c$ are not all of like sign and no one is zero; II. no two of $a, b, c$ have a common odd prime factor; III. either $a, b, c$ are all odd, or two are odd and one is double an odd; IV. - bc, - ac, - ab are quadratic residues of $a, b, c$, respectively.

We shall first prove that I-IV are necessary conditions. Let therefore $f$ represent all integers. It is well known that I follows readily.

If $a$ and $b$ are divisible by the odd prime $p, f$ represents only $1+\frac{1}{2}(p-1)$ incongruent residues $c z^{2}$ modulo $p$. This proves II.

Next, no one of $a, b, c$ is divisible by 8 . Let $a \equiv 0(\bmod 8)$. Every square is $\equiv 0,1$, or $4(\bmod 8)$. First, let $b=2 B$. Since $f$ represents odd integers, $c$ is odd. Since $b y^{2} \equiv 0$ or $2 B$ $(\bmod 8)$ and $c z^{2} \equiv 0, c$, or $4 c, f$ has at most six residues modulo 8. If $m$ is a missing residue, $f$ represents no $m+p n$. Second let $b$ and $c$ be odd. Then $4 b \equiv 4 c \equiv 4(\bmod 8)$. Thus the residues of $f$ modulo 8 are obtained by adding each of 0,4 , $b$ to each of $0,4, c$; we get only seven residues $0,4, b, c$, $4+b, 4+c, b+c$.

No one of $a, b, c$ is divisible by 4 . Let $a$ be divisible by 4 . Since $a$ is not divisible by $8, a \equiv 4(\bmod 8)$. Evidently $f \equiv 0, b, c$, or $b+c(\bmod 4)$. No two of these are congruent modulo 4 . If $b \equiv \pm 1(\bmod 4)$, they are $0, \pm 1, c, c \pm 1$. Evidently $c$ is not congruent to $0, \pm 1$, or $\mp 1$. Hence $c \equiv 2(\bmod 4)$. Since $b \neq 0$, this proves that one of $b$ and $c$ is $\equiv 2(\bmod 4)$. By symmetry, we may take $b \equiv 2(\bmod 4)$. If $b \equiv 6(\bmod 8)$, we apply our discussion to $-f$ instead of 
$f$. Hence take $b \equiv 2(\bmod 8)$. Thus $a \equiv 8 n+4, b=8 m+2$, and $c$ is odd. Since $x^{2} \equiv 0$ or $1(\bmod 4), a x^{2} \equiv 0$ or $8 n+4$ $(\bmod 16)$. Since $y^{2} \equiv 0,1$ or $4(\bmod 8), b y^{2} \equiv 0,8 m+2$, or 8 $(\bmod 16)$. We employ only even residues of $f$ modulo 16 . Then $z$ is even, and $c z^{2} \equiv 0$ or $4 c(\bmod 16)$. But $c \equiv \pm 1$ $(\bmod 4), 4 c \equiv \pm 4(\bmod 16)$. Evidently $a x^{2}+b y^{2}$ has at most $2 \times 3$ residues modulo 16 . The missing two even residues are seen to be $s$ and $s+4$, where $s=10$ if $n$ and $m$ are both even, $s=2$ if $n$ is even and $m$ odd, $s=6$ if $n$ is odd and $m$ even, $s=14$ if $n$ and $m$ are both odd. According as $4 c \equiv 4$ or $-4, f$ is not congruent to $s+4$ or $s$ modulo 16 .

No two of $a, b, c$ are even. Let us set $a=2 A, b=2 B$. By the preceding result, $A$ and $B$ are odd. Also, $c$ is odd. If $A=4 n-1$, we use $-f$ in place of $f$. Hence let $A=4 n+1$. Then $f \equiv 2 x^{2}+2 B y^{2}+c z^{2}(\bmod 8)$. Consider only odd residues of $f$. Then $c z^{2} \equiv c(\bmod 8)$. The residues of $2 x^{2}+2 B y^{2}$ are $0,2,2 B, 2 B+2$. When these are increased by $c$, the sums must give the four odd residues modulo 8 . Hence no two are congruent. Thus no two of $0,1, B, B+1$ are congruent modulo 4 . Since $B$ is odd and $\neq \equiv 1(\bmod 4), B \equiv 3, B+1 \equiv 0$, a contradiction.

This completes the proof of property III. Properties II and III imply the following property.

V. $a, b, c$ are relatively prime in pairs.

Thus $c d \equiv-b(\bmod a)$ has a solution $d$ which is prime to $a$. Suppose that $d$ were a quadratic non-residue of an odd prime factor $p$ of $a$. Write $a=p A$. Consider values of $x, y, z$ for which $f$ is divisible by $p$. Then $z^{2} \equiv d y^{2}(\bmod p)$, whence $y$ and $z$ are divisible by $p$. Hence $f=p F$, where $F \equiv A x^{2}$ $(\bmod p)$. Evidently $A x^{2}$ takes at most $1+\frac{1}{2}(p-1)$ values incongruent modulo $p$. Hence there is an integer $N$ that is not congruent to one of them. Thus $f$ fails to represent $p(N+p w)$ for any value of $w$. This contradiction proves that $v^{2} \equiv d(\bmod p)$ is solvable. The usual induction shows that it is solvable modulo $p^{n}$. Also, $d^{2} \equiv d(\bmod 2)$. By means of the Chinese remainder theorem, we see that $w^{2} \equiv d(\bmod a$, is solvable whether $a$ is odd or double an odd integer, 
Then $w$ is prime to $a$ since $d$ is. Since $(c w)^{2} \equiv-b c(\bmod a)$ this proves IV.

We shall now prove that I-IV imply that $f$ represents every integer $g$. It is known* that I, IV and V imply that $f=0$ has solutions $x^{\prime}, y^{\prime}, z^{\prime}$ which are relatively prime in pairs. Then the greatest common divisor of the three numbers $\alpha=a x^{\prime}, \beta=b y^{\prime}, \gamma=c z^{\prime}$ is 1 . For, if they are all divisible by a prime $p$, one of $x^{\prime}, y^{\prime}, z^{\prime}$ is divisible by $p$ (otherwise $a, b, c$ would all be divisble by $p$ ). By symmetry, let $x^{\prime}$ be divisble by $p$. Then neither $y^{\prime}$ nor $z^{\prime}$ is divisible by $p$. Hence $b$ and $c$ would be divisble by $p$, contrary to V. Hence $\dagger$ if $D$ is any given integer, $\xi, \eta, \zeta$ may be chosen so that

$$
\alpha \xi+\beta \eta+\gamma \xi=D .
$$

We seek a solution of $f=g$ of the form

$$
x=n x^{\prime}+\xi, \quad y=n y^{\prime}+\eta, \quad z=n z^{\prime}+\zeta .
$$

Since $a x^{\prime 2}+\cdots=0, f=g$ is satisfied if

$$
2 D n=g-e,
$$

where

$$
e=a \xi^{2}+b \eta^{2}+c \zeta^{2} .
$$

If $\xi^{\prime}, \eta^{\prime}, \zeta^{\prime}$ is a second set of solutions of (1), write

Then

$$
X=\xi-\xi^{\prime}, \quad Y=\eta-\eta^{\prime}, \quad Z=\zeta-\zeta^{\prime} .
$$

$$
\alpha X+\beta Y+\gamma Z=0 .
$$

We seek the general solution of (5). Let $\delta$ be the greatest common divisor of $\alpha=\delta A$ and $\beta=\delta B$. Then $\delta$ is prime to $\gamma$, whence $Z=-\delta w$. Hence

$$
A X+B Y=\gamma w, A, B \text { relatively prime. }
$$

There exist integers $r, s$ satisfying

$$
A r+B s=1 \text {. }
$$

* Dirichlet-Dedekind, Zahlentheorie, ed. 4, §157, p. 432 (Supplement X).

$\dagger$ Since the g. c. d. 1 of $\alpha, \beta, \gamma$, is a linear function of them. Multiply the relation by $D$. 
Multiply the second member of (6) by (7). Thus

$$
A(X-\gamma r w)+B(Y-\gamma s w)=0 .
$$

The quantities in parenthesis are equal to $B m$ and $-A m$, where $m$ is an integer. The resulting values of $X$ and $Y$, together with $Z=-\delta w$, give the general solution of (6). Hence if $\xi^{\prime}, \eta^{\prime}, \zeta^{\prime}$ is one solution of (1), the general solution is

(8) $\xi=\xi^{\prime}+\gamma r w+B m, \eta=\eta^{\prime}+\gamma s w-A m, \zeta=\zeta^{\prime}-\delta w$,

where $w$ and $m$ are arbitrary, while $r, s$ satisfy (7).

First, let $a, b, c$ be all odd. Then $x^{\prime}+y^{\prime}+z^{\prime} \equiv 0(\bmod 2)$. But $x^{\prime}, y^{\prime}, z^{\prime}$ are not all even. Hence just one of them is even. By symmetry, we may take $x^{\prime}$ even, $y^{\prime}$ and $z^{\prime}$ odd. Then $\alpha$ is even, $\beta$ and $\gamma$ are odd, $\delta$ is odd, $A$ is even, $B$ is odd. Write

$$
e^{\prime}=a{\xi^{\prime 2}}^{2}+b{\eta^{\prime 2}}^{2}+c \zeta^{\prime 2}
$$

When working modulo 2, we may discard the exponents 2 in (4) and (9). Take $w=0, m=1$. Then, by (8),

$$
\begin{aligned}
& \xi \equiv \xi^{\prime}+1, \quad \eta \equiv \eta^{\prime}, \quad \zeta \equiv \zeta^{\prime}, \quad(\bmod 2) \\
& e \equiv \xi+\eta+\zeta \equiv e^{\prime}+1
\end{aligned}
$$

For $w=m=0$, evidently $e=e^{\prime}$. Hence we may take $e \equiv g$ (mod 2). We may take $D=1$. Then (3) yields an integral value of $n$. Hence $f=g$ is solvable.

Second, let $a$ and $b$ be odd, but $c$ the double of an odd integer, whence $c \equiv 2(\bmod 4) . \quad$ Since $f \equiv x+y(\bmod 2)$, $x^{\prime}+y^{\prime}$ is even. But $x^{\prime}$ and $y^{\prime}$ are relatively prime. Hence $x^{\prime}$ and $y^{\prime}$ are odd. Thus $\alpha$ and $\beta$ are odd, $\gamma$ is even, $\delta$ is odd, $A$ and $B$ are odd. By (1) and (4),

$$
D \equiv \xi+\eta \equiv e,
$$

$(\bmod 2)$.

If $g$ is odd, we take $D=1$. (See footnote on p. 59.) By (10), $g-e$ is even and (3) yields an integer $n$. 
But if $g$ is even, we take* $D=2$. By (10), $\xi+\eta$ and $e$ are even. In (8), take $w=0, m=1$. Then

$$
\xi=\xi^{\prime}+B, \quad \eta=\eta^{\prime}-A, \quad \zeta=\zeta^{\prime} .
$$

In case $\xi^{\prime}$ and $\eta^{\prime}$ are odd, we replace $\xi^{\prime}, \eta^{\prime}, \zeta^{\prime}$ by the preceding solution having $\xi$ and $\eta$ even. Hence we may choose the initial solution $\xi^{\prime}, \eta^{\prime}, \zeta^{\prime}$ so that $\xi^{\prime}$ and $\eta^{\prime}$ are even. Then (11) gives

$$
e \equiv e^{\prime}+a B^{2}+b A^{2} \equiv e^{\prime}+a+b \quad(\bmod 4) .
$$

Hence if $a+b \equiv 2(\bmod 4)$, we may choose $e$ so that $e \equiv g$ (mod 4$)$. Then (3) yields an integral value of $n$. But if $a+b \equiv 0(\bmod 4)$, we take $w=1, m=0$ in (8) and see that $\xi$ and $\eta$ are even since $\gamma$ is even. Then since $\delta$ is odd and $c \equiv 2(\bmod 4)$,

$$
e \equiv 2 \zeta^{2}=2\left(\zeta^{\prime}-\delta\right)^{2} \equiv 2 \zeta^{\prime 2}+2=e^{\prime}+2(\bmod 4) .
$$

As before, $f=g$ is solvable.

Corollary. If $a x^{2}+b y^{2}+c z^{2}$ is not a Null form, it does not represent all integers.

Examples with $a=1, c=-C, C>0$.

(i) $b=1$. Then $C$ must be odd or double an odd integer and -1 must be a quadratic residue of $C$. Then every odd prime factor of $C$ is $\equiv 1(\bmod 4)$. A necessary and sufficient condition on $C$ is that it be a sum of two relatively prime squares.

(ii) $b=2$. Then $C$ must be odd and -2 a quadratic residue of $C$. Then its prime factors are $\equiv 1$ or $3(\bmod 8)$. A necessary and sufficient condition on $C$ is that it be of the form $r^{2}+2 s^{2}, r$ odd, $r$ and $s$ relatively prime.

(iii) $b=3$. Then $C$ must be odd or double an odd integer, $C$ prime to 3 , while $C$ and -3 must be quadratic residues of each other. Hence every prime factor of $C$ is $\equiv 1(\bmod 6)$. Necessary and sufficient conditions on $C$ are that $C$ be odd and of the form $r^{2}+3 s^{2}$, where $r$ and $s$ are relatively prime.

The University of Chicago

* Elimination of $\xi, \eta, \zeta$ between (1) and (2) gives $\alpha x+\beta y+\gamma z=D$. Here $D \equiv x+y \equiv f(\bmod 2)$. Hence $D \equiv g(\bmod 2)$. 